inequalities. Reducing access to fast-food stores may have greatest impact for low-income households but mostly in affluent areas. This may imply a greater range of alternatives to fast food in those areas.

\section{OP106 THE CONTRIBUTION OF MATERNAL PSYCHOLOGICAL DISTRESS TO INEQUALITIES IN CHILD MENTAL HEALTH PROBLEMS: DIFFERENTIAL EXPOSURE AND DIFFERENTIAL SUSCEPTIBILITY IN THE UK MILLENNIUM COHORT STUDY}

${ }^{1} \mathrm{~A}$ Pearce ${ }^{*},{ }^{1} \mathrm{PM}$ Henery, ${ }^{1} \mathrm{M}$ Green, ${ }^{2} \mathrm{~S}$ Hope, ${ }^{3} \mathrm{D}$ Taylor-Robinson, ${ }^{1} \mathrm{~A}$ Leyland. ${ }^{1} \mathrm{MRC} / \mathrm{CSO}$ Social and Public Health Sciences Unit, University of Glasgow, Glasgow, UK; ${ }^{2}$ UCL GOS Institute of Child Health, UCL, London, UK; ${ }^{3}$ Department of Public Health and Policy, Institute of Psychology, Health and Society, University of Liverpool, Liverpool, UK

\subsection{6/jech-2019-SSMabstracts. 109}

Background Reducing prevalence and inequalities in child mental health problems (CMHP) will improve life chances and prevent the perpetuation of social inequalities. Research seeking to identify options for reducing health inequalities often aims to identify amenable risk factors on the pathway between socio-economic circumstance (SECs) and health. Such risk factors may create health inequalities via two mechanisms: differential exposure (risk factor is more prevalent in disadvantaged groups) and differential susceptibility (health impacts of the risk factor are worse in disadvantaged groups). Most research focusses on differential exposure, using mediation analysis. However, most mediation methods produce inaccurate estimates if differential susceptibility (i.e. an interaction) is present. Furthermore, differential exposure and differential susceptibility may reveal alternative or complementary policy actions. We used a novel effect decomposition method to examine the contribution of maternal psychological distress (MPD) to inequalities in CMHP in the nationally-representative UK Millennium Cohort Study (born 2000-2002, $n \sim 18,000$ ).

Methods SECs (exposure) were represented by maternal education (GCSE grades $\mathrm{A} * \mathrm{C}$, yes/no, age 9 months), MPD (risk factor) by the Kessler scale (continuous, 3y), and CMHP (outcome) by the Strengths and Difficulties Questionnaire (borderline-abnormal, yes/no, 5y). The total effect (TE) of SECs on CMHP was estimated using risk ratios (RR) and decomposed, using Stata's 'Med4way', into: the direct effect and effects via MPD due to: mediation (differential exposure), interaction (differential susceptibility), and mediated interaction (when SECs affect MPD and its impact on CMHP). Confidence intervals were estimated using non-parametric bootstrapping (1000 replications). We adjusted for baseline confounders (ethnicity, maternal age at first live birth) in a complete case sample $(n=9,777)$. Sensitivity analyses examined bias from unmeasured intermediate confounding and attrition.

Results 10\% children had borderline-abnormal CMH. The RR for the TE of SECs on CMHP was 1.79(1.58-2.04). Two thirds $(66 \%$ [0.50-0.81]) of this was direct, i.e. not acting through MPD. Four percent (1.2-6.8\%) was mediated (differential exposure), $28 \%(12.1-42.2 \%)$ was due to interaction (differential susceptibility), and 2\% (0.6-4.3\%) was from mediated interaction. Bias from unmeasured intermediate confounding and attrition appeared minimal.

Conclusion The direct effect of SECs on CMHP was large. Maternal psychological distress is also potentially important in the development of inequalities, predominantly due to differential susceptibility (and not differential exposure). Thus analyses which only consider mediating pathways may underestimate its role. Policies to improve MPD have the potential to reduce inequalities in CMHP. Future research to understand the factors that buffer advantaged mothers from the consequences of MPD may inform policy content and delivery.

\section{OP107 HOW CAN WE MEASURE COMMUNITY RESILIENCE FOR POPULATION HEALTH? AN EVIDENCE SYNTHESIS FOR WHO EUROPE HEATH EQUITY NETWORK}

1,2 J South*, ${ }^{1} \mathrm{R}$ Jones, ${ }^{1,2} \mathrm{~J}$ Stansfield, ${ }^{1} \mathrm{AM}$ Bagnall. 'School of Health and Community Studies, Leeds Beckett University, Leeds, UK; ${ }^{2}$ Health Improvment Directorate, Public Health England, London, UK

\subsection{6/jech-2019-SSMabstracts. 110}

Background Resilience is a dynamic process of coping, adaptation and growth in response to threats and can be an attribute of individuals, communities or systems. 'Creating resilient communities' is a Health 2020 priority, reflecting the importance of the social environment as a determinant of population health. This is an emergent field for research, with questions of how best to define and measure this complex concept at a community level. This presentation reports on a rapid review and synthesis of measurement strategies conducted for WHO Europe Health Evidence Network (HEN). The review question was: 'What quantitative and qualitative methods can be used to measure health-related community resilience at a national level?'

Methods The rapid review used HEN Evidence Synthesis guidance. A systematic search of academic and grey literature databases and 73 websites combined key terms for community resilience and measurement. Included languages were English, French and German, and Russian (via an independent search). Study selection was in 2 phases, with an initial focus on Europe. Inclusion criteria were articles that reported outcomes involving measurement of health-related community resilience in all population groups, study designs and settings. Studies on individual/system resilience and those not specifying health and wellbeing measurement were excluded. Data extraction fields included theoretical framework, methods and indicators. Findings were summarised in tables and a narrative synthesis. Results In total, 3,753 publications were identified and following screening, 33 studies were included; 27 from WHO European Region. The map of literature showed various measurement frameworks in use, however most related to community disaster resilience. We grouped measurement strategies into:

i. Frameworks providing population profiles of resilience factors, using quantitative data

ii. Mixed method assessments incorporating stakeholder views, used mostly for local planning \& evaluation

iii. Qualitative and participatory approaches, which involved marginalised communities.

There was a dearth of validated measures and insufficient evidence on national-level indicators, but socioeconomic measurement domains were categorised. Key methodological challenges were highlighted, including definitional issues, data aggregation and lack of attention to equity. Notwithstanding these challenges, the review identified some common principles for measuring community resilience. 
Conclusion This review contributes to new understandings of health-related community resilience and its measurement. Using rapid review methods limited the scope of the search, and the focus was mainly on European literature. While there is learning from community disaster resilience methods, transferability to population health needs to be tested.Based on review gaps, recommendations are made for future research topics.

\section{Methodological Issues}

\section{OP112 USE OF OUTCOME 'CHANGE-SCORES' IN OBSERVATIONAL DATA ARE A POTENTIAL SOURCE OF INFERENTIAL BIAS}

\begin{abstract}
1,2,3PWG Tennant, ${ }^{1,2} \mathrm{KF}$ Arnold* ${ }^{1,2} \mathrm{GTH}$ Ellison, ${ }^{4} \mathrm{~J}$ Textor, ${ }^{1,5} \mathrm{SC}$ Gadd, ${ }^{1,2}$ L Berrie, ${ }^{2} \mathrm{~J}$ Ellis, ${ }^{1,2,3}$ MS Gilthorpe. 'Leeds Institute for Data Analytics, University of Leeds, Leeds, UK; ${ }^{2}$ School of Medicine, University of Leeds, Leeds, UK; ${ }^{3}$ The Alan Turing Institute, London, UK; ${ }^{4}$ Department of Tumour Immunology, Radboud University Medical Center, Nijmegen, The Netherlands; ${ }^{5}$ School of Geography, University of Leeds, Leeds, UK
\end{abstract}

\subsection{6/jech-2019-SSMabstracts.111}

Background Studies of change are a cornerstone of research in the health sciences. Robust analyses of change are however extremely challenging, especially in observational data.

In simple exposure-outcome scenarios, one common approach is to create and analyse an outcome 'change-score' by subtracting the baseline outcome from follow-up outcome. Tens-of-thousands of articles can be found that have adopted this approach. Unfortunately, this approach fails to capture the (desired) modifiable component of the outcome variable that occurred after baseline. On the contrary, it retains signreversed information from the baseline outcome that can create extremely-misleading associations.

Using directed acyclic graphs (DAGs) and illustrative simulations, this study explains why outcome change-scores do not capture the true causal quantity of interest and demonstrates the extent of disagreement between robust analyses and change-score analyses in various circumstances.

Methods DAGs with deterministic nodes are used to explain why change-scores do not capture the (desired) modifiable component of the outcome that occurs after baseline. The implications are then illustrated in simulated data, by analysing outcome change-scores with respect to a baseline exposure under several causal scenarios.

Data were simulated using DAGitty $\mathrm{R} 0.2-2$ to match three broad scenarios, with the baseline outcome as 1) competing exposure, 2) confounder, and 3) mediator for the total causal effect of the exposure on the follow-up outcome. Means, standard deviations, and distributions were informed by data from the US National Health and Nutrition Examination Survey for 2009-2014. The association between the baseline exposure and outcome change-score was estimated by linear regression; and the coefficients compared to the known truth and coefficients obtained from robust analyses.

Results Naïve regression analyses of the outcome change-score (insulin) with respect to the baseline exposure (waist circumference) produced biased causal inferences in all scenarios except where the exposure and outcome were uncorrelated at baseline (as in a randomised experiment). When the baseline outcome (insulin) confounded the effect of the baseline exposure (waist circumference) on the follow-up outcome, the naïve regression estimate remained confounded. When the baseline outcome (insulin) mediated the effect of the baseline exposure (waist circumference) on the follow-up outcome, the naïve regression estimate had the opposite sign to the total causal effect.

Conclusion Analyses ofchange-scores should be avoided in observational health research, as they can produce extremely misleading coefficients. Previous observational studies that have naively analysed and interpreted change-score variables should be viewed with extreme caution and any recommendations revisited.

\section{OP113 SEEKING CAUSAL EXPLANATIONS IN POLICY EVALUATION: AN ASSESSMENT OF APPLYING PROCESS TRACING TO THE BARBADOS SUGAR-SWEETENED BEVERAGE TAX EVALUATION}

M Alvarado*, T Penney, J Adams. Centre for Diet and Activity Research, MRC Epidemiology Unit, University of Cambridge, Cambridge, UK

\subsection{6/jech-2019-SSMabstracts.112}

Background Finding an association between a policy and an effect in an observational study is not enough to prove a causal relationship. Impact evaluations may be strengthened by developing an understanding of the causal explanation(s) behind an association. Here, we assess the feasibility of using process tracing (PT). While PT has been applied to a limited number of programme evaluations, we believe this is the first attempt to apply the method to a public health policy evaluation. Given evidence of a statistical association, can PT be usefully operationalized in a public health policy evaluation?

Methods We used the Barbados sugar-sweetened beverage (SSB) tax as a case study. We previously demonstrated an association between tax introduction and an observed decrease in SSB sales. According to dominant theory, price change is the sole mechanism through which SSB taxes dampen consumer demand. However, SSB taxes may also have a signaling effect, raising awareness and reducing demand. Following PT bestpractice, we developed causal theories, pre-specified the evidence we would expect to find under each theory, operationalized tests to identify this evidence, and assessed the probative value of each test. We assessed prior confidence in both theories and described implications of each test.

Results We identified a range of potential tests (8 tests of the price change only theory, 8 separate tests of the signaling effect). For example, one test of the signaling effect could be an assessment of whether the public's perception of 'good' vs. 'bad' drinks matches the pattern of change observed more than a categorization based on taxed vs. untaxed status. In this example, we propose to use print media to qualitatively identify how 'good' and 'bad' drinks were characterized (i.e. were sodas and juices portrayed differently?) and then use this categorization to re-analyze grocery store sales data using an interrupted time series. If this categorization explains the data more fully than an analysis based on taxed/untaxed status, this test would strongly favor signaling over the price change only hypothesis, making this a test with high probative value. We identified methods and data that could be used to empirically assess each test and assessed each test's probative value.

Conclusion Further work will be needed to empirically conduct and critically assess as many of these tests as possible, prioritizing those with greatest probative value. However, this 